\title{
The effects of blood purification combined with antibiotics on extravascular lung water index, inflammatory factors, and prognosis of patients with severe acute pancreatitis complicated with acute respiratory distress syndrome
}

\author{
Bin-Qiang Xu' ${ }^{1}$, Yu Zhou ${ }^{2}$ \\ ${ }^{1}$ Intensive Care Unit, Suzhou Ninth Hospital Affiliated to Soochow University, Suzhou, China; ${ }^{2}$ Clinical Laboratory, Nantong Sixth People's \\ Hospital (Nantong Hospital Affiliated to Shanghai University), Nantong, China \\ Contributions: (I) Conception and design: Both authors; (II) Administrative support: Y Zhou; (III) Provision of study materials or patients: BQ Xu; (IV) \\ Collection and assembly of data: Both authors; (V) Data analysis and interpretation: Both authors; (VI) Manuscript writing: Both authors; (VII) Final \\ approval of manuscript: Both authors. \\ Correspondence to: Yu Zhou. Clinical Laboratory, Nantong Sixth People's Hospital (Nantong Hospital Affiliated to Shanghai University), No. 500, \\ Yonghe Road, Nantong 226000, China. Email: wxljz2@163.com.
}

Background: To retrospectively analyze the effects of routine treatment and blood purification combined
with antibiotics on the extravascular lung water index (EVLWI), inflammatory factors, and treatment
outcomes in patients with severe acute pancreatitis (SAP) complicated with acute respiratory distress
syndrome (ARDS).

Methods: A total of 131 SAP patients admitted to the intensive care unit of Suzhou Ninth Hospital Affiliated to Soochow University from January 2019 to December 2020 were retrospectively enrolled in this study. Patients were divided into two groups according to the treatment methods. In addition to conventional treatment, 60 patients in group A received continuous blood purification (CBP) treatment and 71 patients in group B did not. The EVLWI, inflammatory factors, remission time of clinical symptoms, curative effect, and patient outcomes were recorded at admission and after 1, 3, 5, and 7 days of treatment.

Results: There was a statistically significant difference in the clinical symptom relief time and the clinical efficacy between the two groups of patients $(\mathrm{P}<0.05)$. The mortality rate of patients in group A was $3.33 \%$, which was significantly lower than the $14 \%$ mortality rate observed in patients in group $\mathrm{B}(\mathrm{P}<0.05)$. The EVLWI, as well as the C-reactive protein (CRP), interleukin (IL)-6, IL-8, and tumor necrosis factor (TNF)- $\alpha$ levels were significantly lower in group A patients compared to group B patients on day 1, 3, and 7 posttreatment $(\mathrm{P}<0.05)$. There was also a significant difference in APACHE II scores between the two groups $(\mathrm{P}<0.05)$. The incidence of adverse reactions in group A was $6.7 \%$, which was significantly lower than the $22.5 \%$ incidence observed in group $\mathrm{B}(\mathrm{P}<0.05)$.

Conclusions: Continuous blood purification combined with antibacterial drugs is safe and has a significant effect on the treatment of SAP patient complicated with ARDS, including effectively relieving clinical symptoms and signs, reducing the level of inflammatory factors, and promoting early disease outcomes.

Keywords: Blood purification; severe acute pancreatitis (SAP); acute respiratory distress syndrome (ARDS); extravascular lung water index (EVLWI)

Submitted Jul 16, 2021. Accepted for publication Sep 01, 2021.

doi: 10.21037/apm-21-2168

View this article at: https://dx.doi.org/10.21037/apm-21-2168

(c) Annals of Palliative Medicine. All rights reserved. 


\section{Introduction}

Acute pancreatitis (AP) is the leading cause of hospitalization in patients with digestive diseases (1). A large-scale epidemiological study in California showed that the incidence of AP was 33-44 per 100,000 people (2), and approximately 200,000 patients with AP are hospitalized in the United States each year (3). About $80 \%$ of AP patients have mild AP without serious complications while the remaining $20 \%$ can manifest serious complications and have a high mortality rate (4). Severe acute pancreatitis (SAP) is one of the most common acute diseases in the intensive care unit (ICU), and is characterized by rapid progression, a multitude of complications, and a high fatality rate. Acute respiratory distress syndrome (ARDS) is often the earliest and most common complication. ARDS is characterized by progressive hypoxemia and dyspnea. The incidence of SAP complicated by ARDS is as high as $15-60 \%$, and the fatality rate can reach $60 \%$ within 1 week (5). Patients with ARDS have a high risk of pulmonary infection which further aggravates the disease progression, leading to poor clinical prognosis and a significant increase in patient mortality (6). Continuous blood purification (CBP), also known as continuous renal replacement therapy (CRRT), is one form of treatment for patients with SAP. It works by simulating the glomerular filtration principle of the human body, filtering the patient's blood through a semi-permeable membrane, thereby removing diseases-causing factors such as inflammatory factors and cytokines in the blood. The substances required by the body are reinfused into the blood through replacement. This achieves the purpose of reducing inflammatory reactions, purifying the blood, treating related diseases, and reducing mortality (7). With advancements in this technology, it has been widely used for a variety of clinical diseases, including poisoning, infection, trauma, burns, and multiple organ dysfunction syndrome (MODS) $(8,9)$.

To further improve the outcomes of patients with SAP and ARDS, the clinical efficacy of blood purification combined with antibacterial drugs was investigated. The effects on the EVLWI, inflammatory factors, clinical symptom relief time, curative effect, and patient outcomes were evaluated.

We present the following article in accordance with the STROBE reporting checklist (available at https://dx.doi. org/10.21037/apm-21-2168).

\section{Methods}

\section{Research subjects}

A total of 224 patients with SAP complicated with ARDS who were admitted to the ICU of Suzhou Ninth Hospital Affiliated to Soochow University from January 1, 2018 to January 1, 2021 were retrospectively enrolled in this study. Patients 18 years and older who were diagnosed with SAP according to Atlanta classification standard (10) and ARDS according to the Berlin classification standard (11), and who presented with an onset time $<48$ hours were included in this study. The following exclusion criteria were applied: (I) patients aged $<18$ years old; (II) patients who presented with mild to moderate AP; (III) patients who presented with pregnancy pancreatitis; (IV) SAP patients who had undergone surgical debridement and drainage, nature orifice transluminal endoscopic surgery (NOTES), endoscopic retrograde cholangiopancreatography (ERCP), or other interventional or surgical treatments; $(V)$ the ARDS was not caused by SAP; (VI) patients with malignant tumors, hepatitis, chronic liver failure, chronic renal failure, or other diseases; and (VII) patients whose treatment was interrupted due to abandonment.

According to the inclusion and exclusion criteria, 93 cases were eliminated and a total of 131 cases were finally included in this analysis (Figure 1). The patients were divided into two groups according to their treatment methods. All patients received conventional treatment. In addition, 60 patients underwent continuous blood purification (CBP) treatment (group A). The remaining 71 patients did not receive CBP treatment (group B). The study was approved by the Ethics Committee of Suzhou Ninth Hospital Affiliated to Soochow University, China (2019015) and was conducted in accordance with the Declaration of Helsinki (as revised in 2013). Individual consent for this retrospective analysis was waived.

\section{Therapeutic method}

All patients received routine basic treatment including rest therapy of the pancreas such as diet restriction, gastrointestinal decompression, inhibition of pancreatic enzyme excretion; application of preventive antimicrobial agents; pain relieve; spasmolysis and calmness; nutritional support including liquid recovery and water electrolyte 


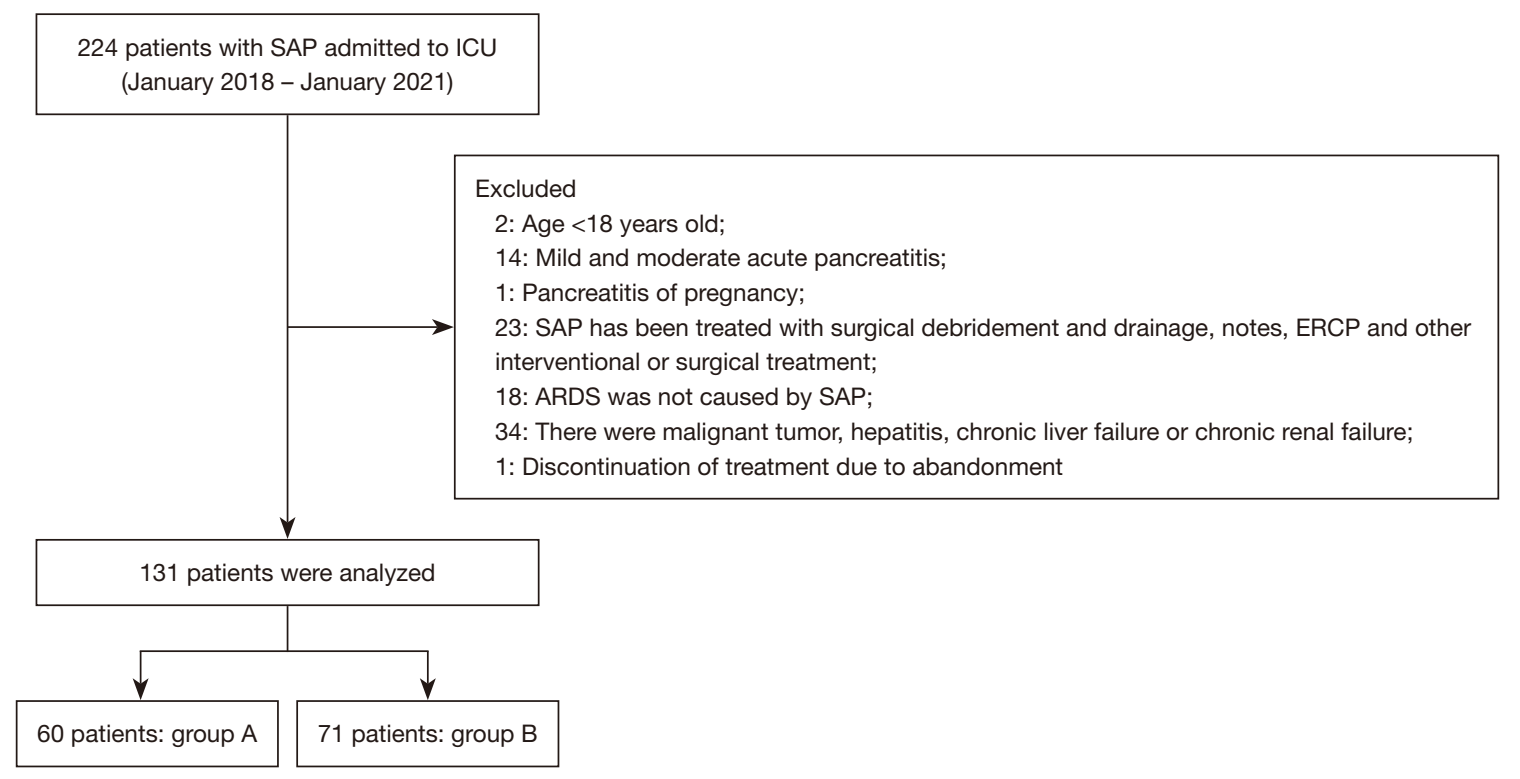

Figure 1 A flow chart showing the patient selection process. SAP, severe acute pancreatitis; ICU, intensive care unit; ERCP, endoscopic retrograde cholangiopancreatography; group A, patients treated with continuous blood purification; group B, patients not treated with continuous blood purification.

balance; and mechanical ventilation treatment.

Patients in the CBP group (group A) also received blood purification treatment. Vascular access was established in patients through deep venous catheters. The Baxter Accura blood purification system (Baxter, USA) with a AV600 polysulfone membrane hemofilter and a membrane area of $1.4 \mathrm{~m}^{2}$ was used. Bicarbonate was used as the replacement fluid. The formula was input using the pre-dilution method, where the replacement fluid volume is $50-100 \mathrm{~mL} \cdot \mathrm{kg}^{-1} \cdot \mathrm{h}^{-1}$ and the blood flow is $200-$ $300 \mathrm{~mL} / \mathrm{min}$.

\section{Observation indexes}

\section{General information}

The basic characteristics of the patients, such as gender, body mass index, age, and cause of disease, were collated.

\section{Disease outcome}

Clinical symptom relief time (such as the relief time of fevers, abdominal pain, and nausea and vomiting) and clinical efficacy and outcome were assessed. The clinical efficacy was calculated as total effective efficiency = apparent efficiency + effective efficiency, and classified as (I) markedly effective, (II) effective, or (III) invalid.

\section{Clinical test indicators}

The EVLWI, as well as inflammatory factors including C-reactive protein (CRP), interleukin (IL)-6, IL-8, and tumor necrosis factor (TNF)- $\alpha$, were assessed prior to treatment and on post-treatment days 1,3 , and 7 . To assess the EVLWI, a three-cavity central venous catheter was inserted into the right subclavian vein, and a $\mathrm{PiCCO}$ arterial catheter was inserted into the left or right femoral artery. The pressure and temperature sensors were connected to the monitor, and $15 \mathrm{~mL}$ of $0-4{ }^{\circ} \mathrm{C}$ normal saline was injected through the central venous catheter to obtain the EVLWI. Other hemodynamic indicators including CRP, IL-6, IL8 , and TNF- $\alpha$ were detected by double antibody sandwich enzyme-linked immunosorbent assays (ELISA; Elabscience, China) in accordance with the manufacturer's instructions.

\section{APACHE II score}

The acute physiology and chronic health score II (APACHE II) was used to evaluate the condition of the two groups before and after treatment. The higher the score, the more serious the condition (12).

\section{Adverse reactions}

The incidence of adverse reactions during treatment was analyzed. 
Table 1 Baseline characteristics of the participants in both groups

\begin{tabular}{|c|c|c|c|c|}
\hline Variable & Group A $(N=60)$ & Group B $(N=71)$ & $t / \chi^{2}$ & $\mathrm{P}$ \\
\hline Gender, n (\%) & & & 1.459 & 0.227 \\
\hline Male & $40(66.7)$ & $40(56.3)$ & & \\
\hline Female & $20(30.3)$ & $31(43.7)$ & & \\
\hline Predisposing factors, $\mathrm{n}(\%)$ & & & 0.936 & 0.919 \\
\hline Biliary diseases & $28(46.7)$ & $34(47.9)$ & & \\
\hline Overeating & $9(15.0)$ & $10(14.1)$ & & \\
\hline Excessive drinking & $13(21.7)$ & $15(21.1)$ & & \\
\hline
\end{tabular}

Group A, patients treated with continuous blood purification; group B, patients not treated with continuous blood purification; BMI, body mass index.

\section{Statistical analysis}

The SPSS 22.0 (SPSS Inc., Chicago, IL, USA) software was used for statistical analysis. Measurement data with a normal distribution was expressed by $\bar{x} \pm s$. The $t$-test of independent samples was used for comparison between groups. The Chi-square test was used for comparison of count data between groups. A P value $<0.05$ was considered statistically significant.

\section{Results}

\section{Basic patient characteristics}

A total of 131 patients were included in this study, of which 60 patients were treated with continuous blood purification (group A), and 71 patients were not (group B). There was no significant difference in gender, age, body mass index (BMI), predisposing factors, nor other general data between the two groups $(\mathrm{P}>0.05$, Table 1$)$.

\section{Disease outcome}

There were statistically significant differences in the clinical symptom relief time between the two groups of patients, as well as the clinical efficacy results at day 3 and day 7 posttreatment $(\mathrm{P}<0.05)$. Most of the patients in both groups improved or recovered and were discharged after treatment. In group A, 58 patients were discharged from hospital and
2 patients died of multiple organ failure on the 14th day of treatment, resulting in a mortality rate of $3.33 \%$. In group B, 61 patients were discharged from the hospital and 10 patients died due to failure of one or more organs after the 13th day of hospitalization, resulting in a mortality rate of $14.1 \%$. The difference in mortality rate between the two groups was statistically significant $(\mathrm{P}<0.05$; Table 2$)$.

\section{Clinical test indicators}

In patients treated with blood purification combined with antibiotics (group A), the EVLWI and levels of CRP, IL6 , IL-8 and TNF- $\alpha$, were significantly lower after 1, 3, and 7 days of treatment compared to patients receiving conventional treatment alone (group B) $(\mathrm{P}<0.05$; Table 3).

\section{APACHE II score}

On days 3 and 7 post-treatment, the APACHE II scores of patients treated with blood purification combined with antibacterial drugs were significantly lower than that of patients who received conventional treatment alone $(\mathrm{P}<0.05$; Table 4).

\section{Adverse reactions}

The overall incidence of adverse reactions in group A was significantly lower than that observed in group B $(6.7 \%$ vs. 
Table 2 A comparison of disease outcomes between the two groups

\begin{tabular}{|c|c|c|c|}
\hline Variable & $\begin{array}{c}\text { Group A } \\
(\mathrm{N}=60)\end{array}$ & $\begin{array}{c}\text { Group B } \\
(\mathrm{N}=71)\end{array}$ & $P$ \\
\hline \multicolumn{4}{|l|}{$\begin{array}{l}\text { Relief time of clinical } \\
\text { symptom (d) }\end{array}$} \\
\hline Nausea/vomiting & $3.28 \pm 1.08$ & $3.63 \pm 1.30$ & 0.099 \\
\hline Abdominal pain & $5.23 \pm 1.45$ & $6.66 \pm 1.77$ & 0.000 \\
\hline Abdominal distension & $5.53 \pm 1.33$ & $6.72 \pm 2.09$ & 0.000 \\
\hline Fever & $3.28 \pm 1.26$ & $5.11 \pm 1.86$ & 0.000 \\
\hline \multicolumn{4}{|l|}{ Clinical efficacy, n (\%) } \\
\hline $3 d$ & & & 0.000 \\
\hline Markedly effective & $23(38.3)$ & $8(11.3)$ & \\
\hline Effective & $19(31.7)$ & $20(28.2)$ & \\
\hline Ineffective & $18(30.0)$ & $43(60.6)$ & \\
\hline Total effective rate & $42(70.0)$ & $28(39.4)$ & \\
\hline $7 d$ & & & 0.000 \\
\hline Markedly effective & $46(76.7)$ & $27(38.0)$ & \\
\hline Effective & $7(11.7)$ & $22(31.0)$ & \\
\hline Ineffective & 7 (11.7) & $22(31.0)$ & \\
\hline Total effective rate & $53(88.3)$ & $49(69.0)$ & \\
\hline Outcome, n (\%) & & & 0.034 \\
\hline Discharged & $58(96.67)$ & $61(85.9)$ & \\
\hline Died of organ failure & $2(3.33)$ & $10(14.1)$ & \\
\hline
\end{tabular}

Group A, patients treated with continuous blood purification; group B, patients not treated with continuous blood purification.

22.5\%; $\mathrm{P}<0.05$; Table 5). After treatment, the incidence of nausea and headaches in group A patients was both $3.3 \%$, which was lower than that experienced by patients in group B (8.5\% and $9.9 \%$, respectively). Edema was not observed in group A patients, while $4.2 \%$ of patients in group B experienced edema.

\section{Discussion}

SAP is an acute critical illness that causes systemic wasting. Inflammation of the pancreas and the release of multiple enzymes results in changes in systemic vascular activities. In addition to the pathological changes of the pancreas itself, dysfunction of multiple organs (MODS) may occur, of which, acute lung injury (ALI) is the most common. The main pathological feature of ALI is pulmonary interstitial
Table 3 A comparison of the clinical data between the two groups

\begin{tabular}{|c|c|c|}
\hline Variable & Group A $(N=60)$ & Group B $(N=71)$ \\
\hline \multicolumn{3}{|c|}{ EVLWI (mL/kg) } \\
\hline $0 \mathrm{~d}$ & $13.98 \pm 2.83$ & $13.61 \pm 2.48$ \\
\hline $1 \mathrm{~d}$ & $12.41 \pm 2.11$ & $13.32 \pm 2.19^{*}$ \\
\hline $3 d$ & $8.22 \pm 1.53$ & $12.54 \pm 1.65^{\star}$ \\
\hline $7 d$ & $5.13 \pm 1.07$ & $10.44 \pm 2.23^{*}$ \\
\hline \multicolumn{3}{|c|}{ CRP (mg/L) } \\
\hline $0 \mathrm{~d}$ & $261.77 \pm 24.82$ & $264.11 \pm 22.43$ \\
\hline $1 d$ & $202.12 \pm 20.02$ & $227.43 \pm 24.82^{*}$ \\
\hline $3 d$ & $183.13 \pm 19.02$ & $192.18 \pm 21.97^{\star}$ \\
\hline $7 d$ & $137.48 \pm 18.33$ & $154.09 \pm 16.84^{*}$ \\
\hline \multicolumn{3}{|c|}{ IL-6 (pg/mL) } \\
\hline $0 \mathrm{~d}$ & $42.71 \pm 6.23$ & $43.03 \pm 5.58$ \\
\hline $1 d$ & $27.7 \pm 3.73$ & $34.97 \pm 5.43^{*}$ \\
\hline $3 d$ & $18.23 \pm 2.79$ & $27.92 \pm 4.99^{\star}$ \\
\hline $7 d$ & $10.32 \pm 3.28$ & $18.95 \pm 3.63^{*}$ \\
\hline \multicolumn{3}{|c|}{ IL-8 (pg/mL) } \\
\hline $0 d$ & $47.36 \pm 10.03$ & $47.24 \pm 10.91$ \\
\hline $1 d$ & $34.53 \pm 8.3$ & $38.75 \pm 7.84^{*}$ \\
\hline $3 d$ & $23.76 \pm 5.82$ & $31.89 \pm 6.22^{*}$ \\
\hline $7 d$ & $15.93 \pm 3.61$ & $23.76 \pm 5.63^{*}$ \\
\hline \multicolumn{3}{|c|}{ TNF- $\alpha(p g / m L)$} \\
\hline $0 \mathrm{~d}$ & $23.71 \pm 4.99$ & $22.49 \pm 5.44$ \\
\hline $1 \mathrm{~d}$ & $15.18 \pm 4.55$ & $18.66 \pm 4.7^{\star}$ \\
\hline $3 d$ & $7.73 \pm 2.67$ & $12.27 \pm 4.03^{*}$ \\
\hline $7 d$ & $2.01 \pm 0.95$ & $6.77 \pm 2.18^{\star}$ \\
\hline
\end{tabular}

Group A, patients treated with continuous blood purification; group B, patients not treated with continuous blood purification; EVLWI, extravascular lung water index; CRP, C-reactive protein; $\mathrm{IL}$, interleukin; TNF, tumor necrosis factor. ${ }^{\star} \mathrm{P}<0.05$ vs. group $\mathrm{A}$.

and alveolar edema caused by pulmonary microvascular endothelial barrier function damage. The EVLWI is used to predict ARDS and facilitates the timely treatment of critically ill patients (13). This retrospective analysis demonstrated that CBP combined with antibacterial drugs effectively decreased the EVLWI, suggesting that the amount of fluid accumulating in the alveolar and pulmonary interstitial space of SAP patients complicated with ARDS 
Table 4 A comparison of APACHE II scores between the two groups $(\bar{x} \pm s)$

\begin{tabular}{lcc}
\hline & \multicolumn{2}{c}{ APACHE II score } \\
\cline { 2 - 3 } & Group A (N=60) & Group B (N=71) \\
\hline 0 d & $18.22 \pm 3.64$ & $17.24 \pm 2.49$ \\
7 d & $10.78 \pm 2.5$ & $13.11 \pm 2.44^{\star}$ \\
\hline
\end{tabular}

Group A, patients treated with continuous blood purification; group $B$, patients not treated with continuous blood purification; APACHE II, acute physiology and chronic health score II. ${ }^{*} \mathrm{P}<0.05$ vs. group $\mathrm{A}$.

Table 5 The incidence of adverse reactions in the two groups of patients

\begin{tabular}{lccc}
\hline Adverse event & $\begin{array}{c}\text { Group A } \\
(\mathrm{N}=60), \mathrm{n}(\%)\end{array}$ & $\begin{array}{c}\text { Group B } \\
(\mathrm{N}=71), \mathrm{n}(\%)\end{array}$ & $\mathrm{P}$ \\
\hline Nausea & $2(3.3)$ & $6(8.5)$ & 0.288 \\
Headache & $2(3.3)$ & $7(9.9)$ & 0.141 \\
Edema & $0(0)$ & $3(4.2)$ & 0.107 \\
Total & $4(6.7)$ & $16(22.5)$ & 0.012 \\
\hline
\end{tabular}

Group A, patients treated with continuous blood purification; group B, patients not treated with continuous blood purification.

can be effectively corrected.

In the early stages of SAP, pancreatic edema and necrosis may occur, which in turn causes local inflammatory cells of the pancreas to release inflammatory mediators into the bloodstream and activate the inflammatory cascade. At the same time, various cytokines and inflammatory mediators including TNF- $\alpha$, IL-6, and CRP, can be detected in the serum of SAP patients. These inflammatory factors are released in large quantities and form a complex network which can damage vascular endothelial cells, increasing their permeability, which in turn causes microcirculation disorders, insufficient bleeding, renal failure, ARDS, and other multiple organ failures. Honore et al. (14) put forward the theory of "immune regulation closure value" which suggests that inflammatory mediators mainly exist in the blood circulation and interstitial spaces, and their levels are in a dynamic state of equilibrium between the two. When inflammatory factors in the circulation are cleared, the inflammatory factors in the interstitial spaces will also be cleared. Once the level of inflammatory factors in the body reaches a certain threshold, the cascade reaction caused by the inflammatory factors will be blocked, thereby reducing damage to the tissues. Blood purification can eliminate the inflammatory factors in the blood circulation through the principles of adsorption, diffusion, and convection (15). Studies have found that CBP can eliminate inflammatory factors in the body, thereby improving immune disorders and promoting the recovery of immune function (16). In the state of advanced immunosuppression, CBP can also strengthen the antigen presentation function of monocytes, thereby improving the body's immune function (17). SAP patients often present with acid-base imbalances, electrolyte imbalances, and blood volume changes. CBP can remove excess body fluids from the body and prevent further loss of plasma, thereby balancing the capacity load. In addition, CBP can also replace fluids according to the patient's condition to promote acid-base balance, improve electrolyte disorders, and maintain a stable internal environment (18). In addition, it is generally believed that SAP is essentially an inflammatory response, so its occurrence and development are always accompanied by the excessive expression of related pro-inflammatory factors (19). Our current research demonstrated that the expression of inflammatory indicators prior to treatment was comparable between group A and group B patients $(\mathrm{P}>0.05)$. However, following treatment, the levels of IL- 6 , IL- 8 , TNF- $\alpha$ and CRP in group A patients were significantly lower compared to group $B$ patients $(\mathrm{P}<0.05)$, suggesting that the combination of antibacterial drugs and continuous blood purification can effectively reduce the body's inflammatory response, block the inflammatory mediator network cascade reaction, and block the process of tissue damage, thereby improving patient prognosis.

First introduced by Knaus in 1981, the APACHE II scoring system is currently the most commonly used and most authoritative method for assessing the severity of critical illnesses in ICU and for predicting patient prognosis. It is simple to calculate and is highly accurate. The higher the APACHE II score, the more severe the patient's condition, and the worse the prognosis (20). In this study, treatment with CBP significantly reduced the APACHEII scores compared to patients in group $\mathrm{B}(\mathrm{P}<0.01)$, suggesting that $\mathrm{CBP}$ can effectively reduce the severity of the disease and improve prognosis.

CBP is relatively safe, with a low incidence of complications. In this study, the incidence of adverse reactions in group A was $6.7 \%$ after treatment, which was significantly lower than the $22.5 \%$ observed with group $\mathrm{B}(\mathrm{P}<0.05)$. This demonstrated that $\mathrm{CBP}$ treatment in patients with SAP is safe and has a low incidence of 
adverse reactions, which aids in the early recovery of patients. However, due to the relatively rapid changes in the condition of patients with AP, it is necessary to closely observe the patient's vital signs during the treatment process, assess the patient's recovery, monitor inflammatory factor levels, evaluate the patient's prognosis, and adjust the treatment plan as required. Improved patient education regarding SAP and CBP will also promote patient compliance and recovery.

\section{Conclusions}

Administration of CBP combined with antibacterial drugs in patients with SAP complicated with ARDS is safe and can effectively relieve clinical symptoms and signs, reduce the level of inflammatory factors, and promote early disease outcomes. However, this study cohort was relatively small and the research content was somewhat limited. Future in-depth studies involving larger multi-center cohorts are warranted to further confirm these results.

\section{Acknowledgments}

Funding: None.

\section{Footnote}

Reporting Checklist: The authors have completed the STROBE reporting checklist. Available at https://dx.doi. org/10.21037/apm-21-2168

Data Sharing Statement: Available at https://dx.doi. org/10.21037/apm-21-2168

Conflicts of Interest: Both authors have completed the ICMJE uniform disclosure form (available at https://dx.doi. org/10.21037/apm-21-2168). The authors have no conflicts of interest to declare.

Ethical Statement: The authors are accountable for all aspects of the work in ensuring that questions related to the accuracy or integrity of any part of the work are appropriately investigated and resolved. The study was approved by the Ethics Committee of Suzhou Ninth Hospital Affiliated to Soochow University, China (2019015) and was conducted in accordance with the Declaration of Helsinki (as revised in 2013). Individual consent for this retrospective analysis was waived.
Open Access Statement: This is an Open Access article distributed in accordance with the Creative Commons Attribution-NonCommercial-NoDerivs 4.0 International License (CC BY-NC-ND 4.0), which permits the noncommercial replication and distribution of the article with the strict proviso that no changes or edits are made and the original work is properly cited (including links to both the formal publication through the relevant DOI and the license). See: https://creativecommons.org/licenses/by-nc-nd/4.0/.

\section{References}

1. Peery AF, Crockett SD, Murphy CC, et al. Burden and Cost of Gastrointestinal, Liver, and Pancreatic Diseases in the United States: Update 2018. Gastroenterology 2019;156:254-272.e11.

2. Frey CF, Zhou H, Harvey DJ, et al. The incidence and case-fatality rates of acute biliary, alcoholic, and idiopathic pancreatitis in California, 1994-2001. Pancreas 2006;33:336-44.

3. Gayam V, Sidhu JS, Mandal A, et al. National Trends and Hospitalizations Related to Pancreatic Cancer in Acute Pancreatitis Patients: A Nationwide Inpatient Sample Study. Cureus 2019;11:e5155.

4. Dong Z, Song J, Ge M, et al. Effectiveness of a multidisciplinary comprehensive intervention model based on the Hospital Elderly Life Program to prevent delirium in patients with severe acute pancreatitis. Ann Palliat Med 2020;9:2221-8.

5. Zhou MT, Chen CS, Chen BC, et al. Acute lung injury and ARDS in acute pancreatitis: mechanisms and potential intervention. World J Gastroenterol 2010;16:2094-9.

6. Riviello ED, Kiviri W, Twagirumugabe T, et al. Hospital Incidence and Outcomes of the Acute Respiratory Distress Syndrome Using the Kigali Modification of the Berlin Definition. Am J Respir Crit Care Med 2016;193:52-9.

7. Hardak E, Avivi I, Berkun L, et al. Polymicrobial pulmonary infection in patients with hematological malignancies: prevalence, co-pathogens, course and outcome. Infection 2016;44:491-7.

8. Liu C, Li M, Cao S, et al. Effects of HV-CRRT on PCT, TNF- $\alpha$, IL-4, IL-6, IL-8 and IL-10 in patients with pancreatitis complicated by acute renal failure. Exp Ther Med 2017;14:3093-7.

9. Li MQ, Shi ZX, Xu JY, et al. Hemodiafiltration combined with resin-mediated absorption as a therapy for hyperlipidemic acute pancreatitis. Cell Biochem Biophys 2014;69:699-702. 
10. Banks PA, Bollen TL, Dervenis C, et al. Classification of acute pancreatitis--2012: revision of the Atlanta classification and definitions by international consensus. Gut 2013;62:102-11.

11. ARDS Definition Task Force; Ranieri VM, Rubenfeld GD, et al. Acute respiratory distress syndrome: the Berlin Definition. JAMA 2012;307:2526-33.

12. Johnson AE, Kramer AA, Clifford GD. A new severity of illness scale using a subset of Acute Physiology And Chronic Health Evaluation data elements shows comparable predictive accuracy. Crit Care Med 2013;41:1711-8.

13. Mojoli F, Bouhemad B, Mongodi S, et al. Lung Ultrasound for Critically Ill Patients. Am J Respir Crit Care Med 2019;199:701-14.

14. Honoré PM, Matson JR. Extracorporeal removal for sepsis: Acting at the tissue level--the beginning of a new era for this treatment modality in septic shock. Crit Care Med 2004;32:896-7.

15. Zheng Z, Qu Y, Ding Y, et al. Research progress on the pathogenesis of acute pancreatitis. Chinese Journal of Hepatobiliary Surgery 2021;27:152-5.

Cite this article as: $\mathrm{Xu} B \mathrm{~B}, \mathrm{Zhou} \mathrm{Y}$. The effects of blood purification combined with antibiotics on extravascular lung water index, inflammatory factors, and prognosis of patients with severe acute pancreatitis complicated with acute respiratory distress syndrome. Ann Palliat Med 2021;10(9):9792-9799. doi: 10.21037/apm-21-2168
16. Zhang Peng, Liu Zhihong, Chen Chaohong, et al. A clinical controlled study of the effect of continuous blood purification on immune homeostasis in patients with severe acute pancreatitis. Journal of Nephrology and Dialysis and Kidney Transplantation 2007:308-15.

17. Peng Z, Singbartl K, Simon P, et al. Blood purification in sepsis: a new paradigm. Contrib Nephrol 2010;165:322-8.

18. Zhang Y, Zeng W, Wang Y, et al. The effect of continuous blood purification in the treatment of severe acute pancreatitis with multiple organ dysfunction syndrome. Journal of Clinical Hepatobiliary Diseases 2016;32:320-3.

19. Yang Z, Dong L, Zhang Y, et al. Prediction of Severe Acute Pancreatitis Using a Decision Tree Model Based on the Revised Atlanta Classification of Acute Pancreatitis. PLoS One 2015;10:e0143486.

20. Zhang XM, Zhang WW, Yu XZ, et al. Comparing the performance of SOFA, TPA combined with SOFA and APACHE-II for predicting ICU mortality in critically ill surgical patients: A secondary analysis. Clin Nutr 2020;39:2902-9.

(English Language Editor: J. Teoh) 